\title{
Los Comentarios Abiertos como Referente de Evaluación de la Docencia Universitaria: La Conveniencia de su Interpretación y Tratamiento
}

\author{
María P. Moreno $^{(1)}$, Martha C. Nava ${ }^{(1)}$ y Margarita Campos ${ }^{(2)}$ \\ (1) Benemérita Universidad Autónoma de Puebla, Facultad de Derecho y Ciencias Sociales, \\ Ciudad Universitaria, Av. San Claudio y 22 Sur, Col. San Manuel, Puebla, Puebla-México. \\ (e-mail: pmr1993@hotmail.com; mar_rojo4@hotmail.com) \\ (2) Benemérita Universidad Autónoma de Puebla, Facultad de Medicina, Av. 13 Sur 2702, Col. Volcanes, \\ Puebla, Puebla, México. (e-mail: armycampos@gmail.com)
}

Recibido Ago. 7, 2013; Aceptado Sep. 9, 2013; Versión final recibida Nov. 6, 2013

\begin{abstract}
Resumen
El propósito de este trabajo es presentar la interpretación y tratamiento de 5734 comentarios abiertos que se registraron a lo largo de 2009 y 2010 como parte del cuestionario institucional en línea de evaluación de la docencia de una Facultad de la Benemérita Universidad Autónoma de Puebla (México). Los comentarios fueron leídos e interpretados para clasificarlos en una tipología de cuatro categorías: ámbito, sobre quién es el comentario, tipo y área universitaria que debe atenderlo. Una vez clasificados, utilizando una base de Excel, los datos se pasaron por un proceso de filtros, para un análisis más detallado. Se muestra que cerca del $95 \%$ de los comentarios corresponden al ámbito académico, acerca del profesor, de los cuales un porcentaje significativo es positivo, y, en menor medida, son negativos y denuncias. Los resultados derivados del análisis de esta información cualitativa es un complemento de la evaluación cuantitativa del cuestionario institucional.
\end{abstract}

Palabras clave: docencia universitaria, evaluación de la docencia, análisis de contenido, comentarios abiertos.

\section{Open Comments as a Reference for the Evaluation of University Teaching: The Benefits of their Interpretation and Analysis}

\begin{abstract}
The purpose of this work is to present the interpretation and analysis of 5734 open commentaries collected during the academic years 2009 to 2010 from the online institutional questionnaire used in the evaluation of teaching in a Faculty at the Benemérita Universidad Autónoma de Puebla (Mexico). The comments were read and interpreted to classify them with a typology consisting of four categories: scope, about whom the comment was made, type and the university department responsible for attending to them. Once classification using Excel was done the data are passed through a process of filters, for a more detailed analysis. The data shows that close to $95 \%$ of the commentaries are about the teacher; a significant percentage is positive, with a low number of negative commentaries and complaints. The analysis of this qualitative information is complementary to the quantitative evaluation of the institutional questionnaire.
\end{abstract}

Keywords: university teaching, teaching evaluation, content analysis, typology, open comments 


\section{INTRODUCCIÓN}

La evaluación de la docencia es una actividad que generalmente toma lugar en las instituciones de educación superior. Desde distintas fuentes se sabe que la docencia presenta sus complejidades para ser evaluada sobre todo si se le asocia a la calidad o eficacia porque no puede ser definida de manera absoluta. Practicada desde el primer tercio del siglo XX, fue en las universidades de Estados Unidos, donde se diseñaron de manera inicial los instrumentos de opinión de estudiantes, mismos que han sido motivo de amplias discusiones sobre todo en lo que se refiere a su diseño y propósito. Reconocido por su trabajo teórico en relación a la evaluación de la docencia, Marsh afirma que la docencia debe evaluarse bajo una concepción multidimensional señalando para ello nueve categorías: a) el aprendizaje y su valor, b) entusiasmo del profesor, c) organización de curso, d) relación satisfactoria, e) interacción del grupo, f) cobertura del curso f) exámenes y evaluación, g) tareas y lecturas y h) carga de trabajo y dificultad. Similares dimensiones fueron el resultado de otros investigadores como Centra y Cashin (Gravestock y Gregor-Greenleaf, 2008). A la fecha, existen otras formas con las cuales se puede evaluar la docencia, entre ellas la autoevaluación y la evaluación de pares. Asimismo, para Arbezú y Gutiérrez (2012) la elaboración de portafolios permite también la reflexión de los alcances de la docencia y sobre todo su profesionalización. De hecho, para Rueda (2010) la formación docente debe responder a un diálogo con los resultados expresados.

Si bien con el paso del tiempo se ha buscado tener más referentes de cómo evaluar el desempeño docente, sigue dominando el uso de cuestionarios de opinión de estudiantes (hoy día incluso en línea), lo anterior, se justifica tomando en consideración las dimensiones poblacionales estudiantiles y docentes que son parte del proceso. Además que los resultados reciben tratamientos estadísticos que permiten entregar resultados al profesorado de manera más expedita. Cabe señalar que es común que dichos cuestionarios de opinión de estudiantes incluyan una sección titulada "Pregunta Abierta" o "Comentarios Adicionales". El propósito de esta investigación es la comprensión e interpretación de los comentarios escritos adicionales.

Para Best (2008) son demasiadas horas las que se invierten cada periodo para evaluar la docencia; los mensajes de los estudiantes deberían servirnos para identificar cómo aprenden y qué expectativas tienen sobre la enseñanza. También señala que los resultados deben ser utilizados de manera sensible. Si bien no todos los cuestionarios de evaluación de la docencia integran una pregunta abierta, en parte, ello responde a que existen diferentes maneras de entender el proceso de evaluación.

Los comentarios adicionales expresan de alguna forma aquello que el estudiante puede manifestar de manera espontánea sin tener como referente el conjunto de reactivos a los que ya ha respondido. En ese sentido, Lewis (1991-92) afirma que existen profesores que demeritan este tipo de comentarios, sin embargo, otros los prefieren sobre los promedios y desviaciones estándar que reciben. Quizá la dificultad de los comentarios abiertos es que son reportados fuera de una estructura comprensible, como lo son los resultados de los cuestionarios estandarizados. En la Universidad de Stanford (1997) consideran que al ser reportados fuera de una estructura, los comentarios se perciben contradictorios, la mitad de los estudiantes dicen una cosa y la otra mitad dice lo opuesto, frente a lo anterior, los académicos suelen frustrarse porque no hay manera de satisfacer a alguno.

De ahí que Perlmutter (2011) sugiera evitar ver los comentarios escritos como un popurrí hecho al azar, además, puntualiza que Lewis desde la década de los noventa, subraya la importancia de construir una matriz o tipología que permita la clasificación de lo escrito, lo anterior de alguna manera da orden al caos de tanta información y muestra las tendencias del desempeño del profesorado. Se agrupan los comentarios atendiendo a las fortalezas docentes, así como toma en cuenta las sugerencias de los estudiantes, e incluso agrupar las inconformidades. En algún momento, Lewis propone utilizar una escala de excelente a pésimo para clasificar los comentarios atendiendo a su contenido.

Bursald y Harrison citan a Braskamp (2008) para señalar que los comentarios escritos de los estudiantes tienen menos credibilidad para los profesores, sí éstos son usados para promoverlos (evaluación sumativa), pero no pierden su valor sí se asocian al mejoramiento profesional (evaluación formativa). En la revisión de la literatura pertinente es común encontrar los resultados de los reactivos estandarizados de un cuestionario, pero pocas veces se alude a las interpretaciones de los comentarios abiertos. Resulta difícil comprender que tan valiosa información no sea considerada, especialmente, cuando se puede identificar sí el estudiante está satisfecho o no con el desempeño del profesor y su aprendizaje. Según la investigación de Gravestock y Gregor-Greenleaf (2008) en universidades canadienses y estadounidenses se utilizan ambas evaluaciones, cuantitativas y cualitativas, sin embargo, no siempre son explícitos en cuanto mencionar como administran la sección escrita del cuestionario. En otros casos, sí manifiestan desde el proceso de recolección hasta el tratamiento. Por ejemplo, en la Universidad de Alberta, los estudiantes pueden elegir mecanografiar los comentarios abiertos fuera del proceso de evaluación en línea. En la 
Universidad de Guelph los comentarios deben ser firmados. Se tienen casos donde el instrumento cuantitativo es anónimo y los comentarios abiertos se firman como ocurre en la Universidad de Queen. En algunas instituciones se les muestran sólo a los profesores, sin guardarse en archivos. En algunos casos son leídos por los jefes de departamento.

En el caso de la Benemérita Universidad Autónoma de Puebla (BUAP), el cuestionario de opinión consta de 36 preguntas; 29 integradas en 7 dimensiones que se abocan a evaluar la relación entre estudiantes y profesores, la mediación como referente de una docencia que tiene sustento constructivista, la estrategias de enseñanza de las que se sirve el profesor, la planeación que se visualiza en relación a las actividades docentes, los alcances y equidad de la evaluación, así como valorar la presencia de los ejes transversales que plantea el Modelo Universitario Minerva en los procesos docentes (BUAP,2010). También el diseño incluye 4 preguntas bajo una dimensión llamada cumplimiento institucional que no tiene un peso numérico en relación al resto del cuestionario. La pregunta 34 funciona como pregunta de control, hace referencia a qué calificación en general se le daría al profesor; la pregunta 35 hace referencia a los materiales y técnicas que el profesor utiliza. Finalmente la pregunta 36 tiene como propósito un comentario adicional del estudiante, cualquiera que éste sea.

Según apreciaciones de la Coordinación de Evaluación Académica Institucional (BUAP, 2012) se registran alrededor de 35000 comentarios adicionales cada cuatrimestre. Al igual que otras instituciones, la BUAP coloca en el registro de cada profesor algunos de los comentarios adicionales para que sean leídos una vez que revisan sus resultados periodo a periodo. Sin embargo, el cúmulo de comentarios de alguna manera obligaba a preguntarse: ¿Cuáles son las principales problemáticas que se señalan? ¿Con qué frecuencia son enfatizadas? ¿Los señalamientos escritos corroboran los resultados de corte cuantitativo? ¿Qué utilidad podrían tener?

Es importante señalar que existen dos fuertes razones para realizar este ejercicio de análisis de contenido de los comentarios adicionales. Primera razón, conviene que las Facultades y Escuelas tengan claridad sobre los asuntos que quizá no han sido considerados en los reactivos que integran el cuestionario. Segunda razón, si se les invita a los estudiantes a comentar abiertamente acerca del desempeño del profesor, entonces, dicha información debe utilizarse y buscar la manera de inferir evidencias, especialmente, para la formación docente.

\section{METODOLOGÍA}

Aun cuando esta investigación responde a una metodología combinada, resulta importante subrayar el interés de mantener la intención de lo cualitativo, esto es, investigar con categorías nativas y no con esquemas preestablecidos. Se hizo uso de la técnica de análisis contenido misma que posibilita elaborar inferencias a partir de ciertos datos. Atendiendo a lo afirmado por Krippendorff (1990) los datos son estímulos físicos o vehículos signos, como las marcas negras sobre un papel blanco. Sin embargo, el interés por el significado parte casi siempre de niveles superiores de abstracción, como documentos escritos, películas cinematográficas, diálogos verbales y pinturas, por mencionar sólo algunos casos. El contexto es su medio. Habitualmente el analista está en condiciones de elegir el medio y la manera de conceptualizarlo. De ahí que dicho autor afirme que debe hacerse explícito el contexto con respecto al cual se analizan los datos (en este caso la educación superior de una facultad de la BUAP en México). Si bien los datos aparecen disponibles de una manera directa, el contexto lo construye el analista con el fin de incluir todas las condiciones circundantes posibles, antecedentes coexistentes o consecuentes. Además es oportuno subrayar que la comunicación de los datos al analista es unidireccional. Es imposible manipular la realidad, no se dispone de una retroalimentación correctiva con la fuente que, por razones propias, le suministra información; el analista se ve obligado a estudiar de manera discreta una parte del universo (en este caso sólo se trata de una facultad).

Los comentarios abiertos dieron pauta para generar a posteriori una tipología que no estaba preconcebida y que fuese un instrumento para clasificar los comentarios acumulados de los años 2009 y 2010 de la BUAP. En el análisis de contenido es necesaria una construcción analítica que ofrezca "las reglas de inferencia", es así como esta tipología determinó a través de sus categorías donde debería ubicarse cada comentario abierto. Dicha tipología (matriz) fue construida por los integrantes de la Coordinación de Evaluación Institucional.

La tipología considera cuatro categorías con sus respectivas subclasificaciones. Es claro para quienes nos avocamos a elaborar el presente ejercicio que la tipología puede ser perfectible. Cada categoría tiene una descripción de lo que comprende, y ello ayuda a visualizar que los comentarios pueden ir más allá del desempeño del profesor. El ámbito hace referencia al espacio al que se refiere el comentario, puede ser 
académico, administrativo, combinado (incluir varios al mismo tiempo), de horario, hacia el programa de evaluación mejor conocido como PIEVA o a la relación entre el profesor y el estudiante.

En relación al ámbito académico se consideran todos los comentarios sobre la forma de enseñar del profesor (es buen maestro, enseña bien, no sabe enseñar; también se incluyen características propias del diseño curricular: el programa de la asignatura es muy largo, está mal ubicado en el plan de estudios, se incluyen contenidos que no se entienden. En cuanto al ámbito administrativo hace referencia a los comentarios que se refieran a la mejora y/o deficiencia de la administración, de la infraestructura, inmobiliario, laboratorios y equipo. Siguiendo tal idea, por ejemplo, "los equipos de cómputo no funcionan, o las aulas se encuentran sucias". En el ámbito relacional se incluyan aquéllos comentarios que señalan el tipo de relación que guarda el profesor con los estudiantes, por ejemplo, "es amable en su trato", "es respetuoso", "grosero", "altanero", "impaciente", "intolerante".

En la categoría denominada PIEVA se clasifican los comentarios que aluden al Programa de Evaluación Académica, por ejemplo, "estas encuestas no sirven" o "el cuestionario es muy largo", "no he visto resultados de estas evaluaciones". La categoría horario y asistencia incluyen aquéllos comentarios vinculados a su incumplimiento, es el caso de registrar: "no asiste a clases", "se va antes del horario establecido"," por lo general falta y no avisa". Combinado es una categoría que puede presentar varios comentarios con distintos propósitos, por ejemplo, "es un buen profesor, pero muy altanero" o bien "es un buen profesor, pero el programa es muy largo"

Una vez que se ha interpretado el ámbito, la relectura permite saber sobre quién recae dicho comentario: el profesor, la materia, otros profesores, la escuela, PIEVA o bien en general la Universidad. El contenido del comentario nos permite saber sí es una denuncia. También puede ser un comentario positivo o negativo (sin llegar al nivel de denuncia). Puede ser que el estudiante sólo haga una sugerencia, o esté dando aviso de una suplencia de mutuo propio, esto es que el profesor titular de la materia no llega y envía a otro sin aviso a la dirección. Cuando se habla de combinado es posible que un comentario pueda considerar varios aspectos. Una vez interpretado el comentario varias veces, se puede determinar qué instancia deberá dar solución al problema. Por ejemplo, se puede encontrar un comentario que haga mención al ámbito de horario y sea sobre la materia, además de tipo negativo, y deba ser referido a las instancias administrativas.

Tabla 1: Tipología para clasificar comentarios abiertos

\begin{tabular}{|l|l|l|l|}
\hline Ámbito & Sobre quién & Tipo & Área de Atención \\
\hline Académico & Profesor & Denuncia & Academias \\
\hline Administrativo & Materia & Positivo & Director \\
\hline Combinado & Otros profesores & Negativo & Escuela de Formación Docente \\
\hline Horario & Escuela & Sugerencia & Instancias Administrativas \\
\hline PIEVA & PIEVA & Mutuo propio & \\
\hline Relacional & Universidad & Combinado & Mismo profesor \\
\hline
\end{tabular}

El ejercicio implicó leer cada uno de los 5734 comentarios abiertos y ubicarlos en alguna de las categorías arriba señaladas. Siguiendo las pautas que algunos teóricos señalan para el análisis de contenido se tomó en cuenta que hay dos perspectivas: el "contenido manifiesto es el único dato real" y "el contenido latente debe ser inferido de alguna forma" ésta última defendida por Krippendorff (1990). Se procuró la rigurosidad ante la lectura del contenido, especialmente, si se toma en cuenta que son datos "no estructurados" que se relacionaron con un conjunto de categorías analíticas, evitando forzar el texto. Asimismo, se tiene claro que los estudios cualitativos tienen como limitante su transferencia y generalización de los resultados. Sin embargo, cabe decir que cualquier institución que considere en sus evaluaciones de desempeño docente comentarios abiertos no debe desaprovechar la oportunidad de su interpretación y tratamiento.

Resulta necesario puntualizar que la presente investigación hace referencia a la primera fase de los hallazgos que se han visualizado en razón de la clasificación. Básicamente alude al ámbito académico, es sobre el profesor, en todos los tipos y las instancias que deben atender los señalamientos. El formato Excel permitió tener al final del ejercicio apreciaciones de corte cuantitativo. 


\section{RESULTADOS Y DISCUSIÓN}

La riqueza de los contenidos permite tener claro que el estudiante muestra madurez para hacer señalamientos serios, asunto que podría descartarse si se toma en cuenta que evalúa en línea y existe el anonimato. En cuanto a los hallazgos mencionados, se destaca que los 5734 comentarios abiertos corresponden a 298 profesores de los 301 evaluados de una Facultad de la Benemérita Universidad Autónoma de Puebla. Lo anterior significa que durante seis periodos que son los correspondientes a 2009 y 2010 (primavera, verano, otoño respectivamente) sólo tres profesores no tuvieron comentario alguno. Siguiendo tal idea, se tienen profesores con un sólo comentario en dos años hasta un profesor que se ubica con 118 comentarios abiertos, es el que más ha recibido. De los 5734 comentarios abiertos, 5417 (94.5\%) resultan del cruce entre ámbito académico sobre el profesor en todos sus tipos. Cabría decir que sólo 317 (5.5\%) salen del ámbito del desempeño del profesor, pero no por ello menos importante (ver tabla 2). Algunos aspectos específicos relacionados con los resultados generales mostrados en la Tabla 2, se detallan a continuación:

Tabla 2. Comentarios abiertos correspondientes a 2009-2010 de una Dependencia de Educación Superior.

\begin{tabular}{|l|c|c|c|c|c|c|c|}
\hline Ámbito & \multicolumn{7}{|c|}{ Tipo } \\
\hline Académico-Sobre: maestro & Positivo & Negativo & Sugerencia & Denuncia & Mutuo propio & Combinado & Total \\
\hline & 3617 & 713 & 539 & 28 & 3 & 517 & 5417 \\
\hline Porcentaje & $63.10 \%$ & $12.40 \%$ & $9.40 \%$ & $0.50 \%$ & $0.10 \%$ & $9.00 \%$ & $94.50 \%$ \\
\hline
\end{tabular}

\section{Tipo positivo:}

Comentarios en esta categoría: se hallan 3617 que generalmente evocan al buen desempeño del profesor, incluso, a sus cualidades no sólo docentes, sino a sus cualidades como persona. Dentro de los comentarios abiertos pueden encontrarse algunos que manifiestan el reconocimiento que por años algunos profesores han mantenido. Algunos ejemplos de comentarios positivos: 1) "En lo particular, presenta sus clases de manera muy activa y eso hace que tengamos interés"; 2) "Cumple con todas las características para decir que es una excelente profesor, su método de enseñanza es muy eficiente"; y 3) "Excelente profesor, no hay palabras para describir lo mucho que me ha hecho reflexionar acerca del derecho penal y aprender por mí misma lo que con su metodología me enseña. Ninguno como él. Es el mejor maestro que he tenido en toda mi vida. Gracias por tenerlo en la BUAP"

Dado el número de comentarios abiertos positivos se sugiere, a partir de la tipología, que a todos aquéllos profesores con un número significativo de señalamientos positivos se les haga de su conocimiento. A los profesores les convendría saber que los comentarios abiertos dan referencia de sus logros. Lo anterior se sugiere porque no todo el profesorado consulta sus resultados de evaluación en línea aunque se promueva.

\section{Tipo negativo:}

Comentarios en esta categoría se clasifican 713. Los comentarios principalmente señalan la falta de competencias didácticas de los profesores; algunos ejemplos de comentarios en ese tenor: 1) "La profesora es muy buena pero necesita un empujoncito para aprender a dar bien sus clases porque cuando quiere es muy interesante su clase....."; 2) "Considero que no tiene un buen dominio de su clase, no sabe transmitir sus ideas de una forma clara y termina confundiendo y cae en errores con frecuencia, pero no acepta dichos errores, aunque los materiales que aporta como sus bibliografías hacen que comprendas los temas. Pero entonces considero que valdría más comprar los libros y no tener maestro"; y 3) "Debería de modificar su método de enseñanza pues sólo transcribe en el pizarrón los apuntes que trae y aparte de eso es muy egoísta pues si le preguntamos algo dice que nosotros lo investiguemos aparte". Los comentarios de este bloque han sido referente para saber quiénes son aquéllos profesores que deberían asistir a la Escuela de Formación Docente. Además, de puntualizar que en este categoría se encuentran comentarios de manera persistente sobre algunos profesores.

\section{Tipo sugerencia:}

En relación a los hallazgos en dicha categoría se puede hacer evidente con las 539 sugerencias que el alumno muestra madurez para proponer mejoras que pueden hacer avanzar el desempeño del profesor, y no sólo ello, sino incluso mejorar el aprendizaje, en ese sentido, se expresaron algunos ejemplos que corresponden a distintos profesores como los siguientes: 1 ) "Considero que el profe tiene los conocimientos 
necesarios para enseñar lamentablemente hay ocasiones en que a los alumnos nos faltan algunas bases yo considero que lo ideal sería que quien te dé Derecho Civil I debería continuar con todos los que siguen para darle continuidad a nuestro aprendizaje"; 2) "Muy buena profesora, pero habla muy rápido"; 3) "Sólo debería mejorar la puntualidad"; 4) "Que el maestro conociera a las personas antes de formarse un criterio de ellos, ya que antes de conocerlas clasifica, y para mí eso no es correcto, ya que las apariencias engañan y no tiene porque guiarse sólo por instinto, como él dice"; 5) "Que respete el temario que se ha asignado a la materia y que llegue temprano"; 6) "Pues se me hace que deberían darle vacaciones y mandarla a un curso de redacción porque escribe muy feo, además no tiene buena dicción en sus palabras y según da ejemplos pero te deja en las mismas"; 7) "En general la maestra es buen catedrático, sólo que la mitad del curso se la pasa hablando de ella. A mí parecer es algo que a nosotros como estudiantes no nos interesa. Mientras se dedique a hablar sólo de los temas que le conciernen a la materia sería mejor persona y catedrático"; y 8) "iCon todo respeto, si la profesora ya brindo sus servicios y en su tiempo fueron de buen rendimiento, es mejor retirarse con honor! ¡Ya no nos tiene paciencia, no nos brinda confianza, todo le molesta!"

El abanico de sugerencias indica que la tipología deberá considerar, en un futuro, otras subcategorías que permitan tener más especificidad. En un número significativo se registraron sugerencias en relación a la asistencia y puntualidad del profesorado. Cuestión que amerita ser un asunto referido a los directivos. Y la persistencia en no respetar el programa de la asignatura y hablar de asuntos no referidos al contenido del curso a las academias respectivas.

\section{Tipo mutuo propio:}

En esta categoría fueron suscritos los comentarios abiertos que mencionaban que el profesor titular había dejado el curso por alguna razón, en ciertos casos, con conocimiento de la administración, pero en otros simplemente había enviado a alguna persona de mutuo propio y sin tener las competencias para desarrollar tal trabajo. En ese sentido, fueron pocos los comentarios, 3 (0.1\%).

\section{Tipo denuncia:}

Las denuncias sólo representan un $0.5 \%$ del total de los comentarios, sin embargo, lo anterior no debería ignorarse pues se vinculan principalmente con asuntos delicados entre ellos el acoso y la presencia en estado inconveniente de algunos docentes.

\section{Tipo combinado:}

En este apartado se recuperan $539(9 \%)$ comentarios abiertos, denominados combinados pues en el mismo se identifican varios aspectos, lo que evita ubicarlo en una sola categoría: 1) "Debe poner más atención en el grupo y planear mejor sus clases, ser más puntual y asistir para que sus alumnos no perdamos el interés en su materia, que explique todos los contenidos para que a la hora del examen no pregunte algo que ni idea tenemos de que se trata"; y 2) "El curso fue muy bueno y la verdad me agradó que el maestro supiera tanto pues me alienta para estudiar y aprender cosas de las que yo ni idea tenía que ocurre en nuestro país, sin embargo, redunda mucho en los temas y pierde la ilación de sus comentarios con el tema de la clase".

Lo anterior hace visible que en un mismo texto se puede reconocer dos o tres ideas sobre el desempeño del profesor. De ahí la necesidad de llamar a la categoría combinado.

El análisis de contenido realizado, aunque sólo tuvo finalidades descriptivas, procuró también buscar su validez y confianza de manera indirecta. Por ejemplo, la tipología fue usada en otras facultades donde los resultados se acercaron a los de la facultad aquí estudiada (BUAP, 2012). De igual manera los resultados cuantitativos del cuestionario permitieron identificar cerca de 139 profesores que cumplen con la dimensión formativa que se espera de ellos, y 82 que carecen de los elementos idóneos para realizar la docencia universitaria que los estudiantes señalan . Se advierte que los profesores con altos puntajes en la sección cuantitativa, reciben también un significativo número de comentarios positivos. Fondón, Madero y Sarmiento (2010) refieren las problemáticas que los profesores noveles tienen en el ejercicio de la docencia cuestión que parece coincidir en los comentarios abiertos de la BUAP donde también esto es señalado.

\section{CONCLUSIONES}

Los comentarios abiertos adicionales que se integran a los instrumentos estandarizados para evaluar la docencia deben ser administrados bajo categorías analíticas tanto para su clasificación como para uso institucional, especialmente, cuando son dados a conocer al profesor. Presentar los comentarios abiertos como un popurrí al azar confunde más que ayuda a mejorar la docencia. 
Los comentarios abiertos administrados resultan un complemento de la evaluación cuantitativa pues señalan aquellas fortalezas y debilidades que el estudiante subraya más allá de los ítems que ya contestó en la sección cuantitativa. En ese sentido, se advierten una serie de observaciones que van desde la necesidad de formación pedagógico-didáctica del profesorado así como el desconocimiento incluso de la disciplina que imparte, pasando por fallas curriculares al no cumplir con las actividades del programa. También se hacen señalamientos en el sentido de la falta de cumplimiento en el horario de clase y la asistencia.

La administración sistematizada de los comentarios permite incluso señalar quien debería avocarse a resolver las fallas señaladas, por ejemplo, cuando se trata de incumplimiento en el horario y asistencia del profesorado es el director de la facultad ( es la persona que contrata al profesorado en una facultad) quien debe resolver estas cuestiones, cuando es una sugerencia en relación a la falta de elementos didácticos del profesor, entonces, debe ser referido a la Escuela de Formación Docente. Sin dejar a un lado a las academias de profesores cuando el contenido abordado no es el señalado en el programa. Incluso el mismo profesor debe recibir dicha información sea positiva o negativa.

Aun cuando los comentarios abiertos resulten un complemento de la evaluación cuantitativa, su sistematización orientan en el sentido de tener claridad sobre qué tipo de formación docente es la adecuada para una facultad como la estudiada. Además sí los comentarios son positivos ayudan a modelar un perfil de docente desde la perspectiva de estudiante.

\section{REFERENCIAS}

Álvarez, J. L., Cómo hacer investigación cualitativa. Fundamentos y Metodología, Paidós Educador, México, D.F (2003).

Arbesú M.I.; Gutiérrez, E., El portafolio docente un medio para reflexionar y evaluar las competencias. Revista Iberoamericana de Evaluación Educativa. ISSN: 1989-0397 Vol. 5(2) $52-59$ (2012). http://www.rinace.net/riee/numeros/vol5-num2/art4.pdf Acceso: Julio 22 (2013).

Best, A., Student evaluations of law teaching work well: strongly agree, agree, neutral, disagree, and strongly disagree. Southwestern Law Review. Vol.38 1-36 (2008). https://www.swlaw.edu/pdfs/lr/fall08_best.pdf Acceso: 29 de Julio (2013).

Bursald, Ch. A.; Paul, D. H., Further evidence supporting the validity of both a multidimensional profile and an overall evaluation of teaching effectiveness, Assessment \& Evaluation in Higher Education, Vol. 33, No. 5, 567576 (2008).

BUAP., Cuestionario de evaluación de la docencia 2010. Benemérita Universidad Autónoma de Puebla. México. http://www.evaluación.buap.mx. Acceso: 20 Marzo (2012).

BUAP., Entrevista responsable de Coordinación de Evaluación Académica. Benemérita Universidad Autónoma de Puebla. México. (2012).

Fondón,I.; Madero, M.; Sarmiento, A., Principales Problemas de los Profesores Principiantes en la Enseñanza Universitaria, Formación Universitaria. Vol.3 (2) 21-28 (2010). http://www.citrevistas.cl/a1formacion.htm. Acceso: 10 de Octubre (2013).

Gravestock, P.; Gregor-Greenleaf, E., Student Course Evaluations: Research, Models and Trends. Toronto: Higher Education Quality Council of Ontario. (2008).

http://www.heqco.ca/SiteCollectionDocuments/Student\%20Course\%20Evaluations_Research,\%20Models\% 20and\%20Trends.pdf. Acceso 10 de Octubre (2013).

Krippendorff, K., Metodología de análisis de contenido. Teoría y práctica, Ediciones Paidós, Barcelona, España. (1990).

Lewis, K., Making Sense (and use) of Written Student Comments. Essays on Teaching Excellence. Toward the Best in the Academy. Vol. 3 (8) 1-5 1991-1992

http://www.podnetwork.org/publications/teachingexcellence/91-92/V3,\%20N8\%20Lewis.pdf. Acceso: 29 Julio (2013).

Perlmutter,D., How to read a student evaluation. The Chronicle of Higher Education. 30 Octubre (2011).http://chronicle.com/article/How-to-Read-a-Student/129553/ Acceso: Julio 29 (2013). 
Rueda, M., Reflexiones generales a considerar en el diseño y puesta en operación de programas de evaluación de la docencia. Revista Iberoamericana de Evaluación Educativa. Vol.3 (1) 345-350 (2010). http://www.rinace.net/riee/numeros/vol3-num1_e/reflexiones.pdf Acceso Julio 26 (2013).

Stanford University, Using Student Evaluations to Improve Teaching. Speaking of Teaching. Vol.9 (1) 1- 4 (Otoño 1997).http://www.stanford.edu/dept/CTL/Newsletter/student_evaluations.pdf.Acceso:11de octubre (2103).

Taylor, S.J. y Bogdan, R., Introducción a los métodos cualitativos de investigación. Paidós Básica, Barcelona, España. (1987). 